\title{
A simplification of entanglement purification
}

\author{
Jin-Yuan Hsieh ${ }^{1}$, Che-Ming $\mathrm{Li}^{2}$, and Der-San $\mathrm{Chuu}^{2}$ \\ ${ }^{1}$ Department of Mechanical Engineering, Ming Hsin University \\ of Science and Technology, Hsinchu 30401, Taiwan. \\ ${ }^{2}$ Institute and Department of Electrophysics, National Chiao \\ Tung University, Hsinchu 30050, Taiwan.
}

November 5, 2018

\begin{abstract}
An idea of hybrid maps is proposed to establish standard entanglement purification protocols which guarantee to purify any distillable state to a desired maximally entangled pure state all by the standard purification local operations and classical communications. The protocols proposed in this work, in which two state transformations are used, perform better than the IBM and Oxford protocols in the sense that they requir fewer operation times in yielding a same amount of the desired pure state. One of the proposed protocol in this work can even lead to a higher improved output yield when it is combined with the hashing protocol, as compared with the combined algorithm consisting of the Oxford and the hashing protocol.

PACS:03.67.-a
\end{abstract}

Quantum information processings such as quantum teleportation[1], quantum data compression[2,3], and quantum cryptography[4] rely on the transmission of maximally entangled qubit pairs over quantum channels between a sender(Alice) and a receiver(Bob). The quantum channel, however, is always noisy due to the interaction with the environment and even possibly the measurement controlled by an eavesdropper. Therefore, the pairs shared by Alice and Bob are no longer of the desired pure ones to begin with a quantum processing. The resource in the noisy channel then can be viewed as a mixed state, or equivalently, an ensemble of pure states with definite random probabilities. The probabilities of the pure states in the ensemble are random so should be unknown to Alice and Bob in advance of a quantum processing. Accordingly, Alice and Bob need to take an action of entanglement purification to regain, at least asymptotically, the desired maximally entangled pure state if the mixed state is distillable. This aim can be achieved by Alice and Bob, using consecutive local operations and classical communications (LOCC).

Two typical recurrence methods of entanglement purification should be mentioned. The first entanglement purification protocol (the IBM protocol) was developed by Bennett et. al. $[5,6]$ in achieving a faithful quantum teleportation. Soon later, an improved protocol entitled 
"Quantum Privacy Amplification" (QPA, or the Oxford protocol) was addressed by Deutsch et al.[7] in consideration of the security of a quantum cryptography over noisy channels. Both the IBM and Oxford protocols are capable of purifying a desired maximally entangled pure state from every distillable mixed state whose components are not learned by Alice and Bob initially. By using the IBM protocol, Alice and Bob can asymptotically regain the desired pure state, but they have to consume operation time in twirling the state in between each purification LOCC operation into a Werner state[8] whose fidelity relative to the desired pure state is always greater than $1 / 2$. Compared with the IBM protocol, the Oxford protocol can provide higher output yield, defined as the purified pairs per impure input pair, especially when the initial fidelity with respect to the desired pure state of the input state is close to $1 / 2$. In particular, the Oxford protocol is capable of purifying any state whose average fidelity with respect to at least one maximally entangled pure state is greater than $1 / 2$ and can be directly applied to purify states which are not necessarily of the Werner form. However, since the Oxford protocol occasionally may purify a pure state other than the desired one, i.e., it could yield two possible pure states, depending on the initial mixed state, Alice and Bob then are suggested to take efforts additional to the purification LOCC operations to transform the pure state with greatest component $(>1 / 2)$ in the input mixed state into the desired state; such action also costs operation time in the additional local unitary operations and classical communications to identify the mixed state and thus consumes some pairs before the standard purification LOCC operations. The output yields induced by the IBM and Oxford protocols are rather poor, but can be increased somewhat provided both protocols are combined with hashing protocols, as described in refs. $[5,6]$. So far, there have been modified protocols dedicated to increasing the yield of an entanglement purification procedure, e.g., see refs. [9-11].

Surveying on these modified methods, one finds that while inducing greater yields, they at the same time require more local unitary operations and classical communications in the reordering schemes and hashing protocols $[5,6]$ that are combined in the standard purification protocols. So, when comparing the performances of two protocols, say A and B, we can say protocol A performs better than B either when the yield of protocol A is greater than that of protocol B if both protocols cost equal operation times, or when protocol A requires less operation time than protocol B provided they induced equal yields. Instead of focusing on increasing the yield, in this work we are intended to propose an idea of establishing entanglement purification protocols in which the required operations are the fewest, when compared with the standard IBM and Oxford protocols. These protocols can purify a desired pure state by using the standard LOCC operations alone. When using these protocols, the mixed state to be purified needs not be transformed into the Werner state nor be reordered so that its fidelity with respect to the desired pure state is the largest. Furthermore, the protocols presented in this work in fact can provide better yields than that induced by the Oxford protocol.

The standard purification LOCC operation considered in this work, as shown in Fig. 1, should be mentioned first. In each purification LOCC operation, Alice and Bob first perform local operations by operators $U$ and $U^{*}$, which will be defined latter, respectively. Then Alice and Bob each performs a quantum control-not operation. They then measure the target qubits in the computational basis, and if the outcomes, communicated via classical channel, coincide they keep the control pair for the next step and discard the target pair. If the outcomes do 
not coincide, both pairs are discarded. In the purification LOCC operation, the state to be purified needs not be of a Werner form. We express the mixed state in the Bell basis $\left\{\left|\Phi^{+}\right\rangle\right.$, $\left.\left|\Psi^{-}\right\rangle,\left|\Psi^{+}\right\rangle,\left|\Phi^{-}\right\rangle\right\}$:

$$
\begin{aligned}
& \left|\Phi^{ \pm}\right\rangle=\frac{1}{\sqrt{2}}(|00\rangle \pm|11\rangle), \\
& \left|\Psi^{ \pm}\right\rangle=\frac{1}{\sqrt{2}}(|01\rangle \pm|10\rangle),
\end{aligned}
$$

where $|0\rangle$ and $|1\rangle$ form the computational basis of the two-dimensional space belonging to the EPR pairs. Let $\left\{a_{0}, b_{0}, c_{0}, d_{0}\right\}$ be the average initial diagonal elements of the density operator representing the mixed state before the protocol is begun with, and $\left\{a_{r}, b_{r}, c_{r}, d_{r}\right\}$ be the average diagonal elements of the surviving state after the $r$-th step. It can be shown that a purification LOCC operation in fact is relative to a nonlinear map, where the diagonal entries of the surviving state after the LOCC operation are nonlinear functions of those before the operation. Therefore the purification protocol considered in this work is composed of consecutive nonlinear maps of the Bell-diagonal elements used to transform an initial state asymptotically to a desired pure state. Suppose the state $\left|\Phi^{+}\right\rangle\left\langle\Phi^{+}\right|$is the desired one to be purified through the purification, we then are willing to map step by step the initial state $\left\{a_{0}\right.$, $\left.b_{0}, c_{0}, d_{0}\right\}$, where one of the elements should be greater than $1 / 2$, to converge to the desired attractor $\{1,0,0,0\}$ as the step number $r$ is sufficiently large. But the intrinsic property of the nonlinear map reveals that the desired attractor is not the only one, as can be seen in the article of Macchiavello[12], who has given the analytical convergence in the recurrence scheme of the QPA protocol. The interesting nonlinear behavior of the recurrence scheme in a distillation protocol is dominantly influenced by the local unitary operations operators $U$ and $U^{*}$ applied by Alice and Bob in the purification LOCC operation. Generalized expression for $U$, controlled by two phases $\theta$ and $\phi$, is given by

$$
U(\theta, \phi)=\left[\begin{array}{cc}
\cos \left(\frac{\theta}{2}\right) & -e^{-i \phi} \sin \left(\frac{\theta}{2}\right) \\
e^{i \phi} \sin \left(\frac{\theta}{2}\right) & \cos \left(\frac{\theta}{2}\right)
\end{array}\right] .
$$

It is clear that distinct choices of $\theta$ and $\phi$ will lead to different destinations of the protocol. For example, in using the original QPA protocol, Alice and Bob choose $\theta=\phi=\pi / 2$, i.e., they apply the operator

$$
U\left(\frac{\pi}{2}, \frac{\pi}{2}\right)=\frac{1}{\sqrt{2}}\left[\begin{array}{cc}
1 & -i \\
i & 1
\end{array}\right] .
$$

In this case, one will have a map $\left\{a_{r-1}, b_{r-1}, c_{r-1}, d_{r-1}\right\} \rightarrow\left\{a_{r}, b_{r}, c_{r}, d_{r}\right\}$ according to the following relations:

$$
\begin{aligned}
& a_{r}=\frac{a_{r-1}^{2}+b_{r-1}^{2}}{p_{r-1}}, b_{r}=\frac{2 c_{r-1} d_{r-1}}{p_{r-1}}, \\
& c_{r}=\frac{c_{r-1}^{2}+d_{r-1}^{2}}{p_{r-1}}, d_{r}=\frac{2 a_{r-1} b_{r-1}}{p_{r-1}}, \text { for } \theta=\phi=\pi / 2,
\end{aligned}
$$


Figure 1: The standard purification LOCC operations including the local controlled-NOT operation, single qubit measurement, and local unitary operation in each party. Note that the classical communication is not shown in this figure.

where $p_{r-1}=\left(a_{r-1}+b_{r-1}\right)^{2}+\left(c_{r-1}+d_{r-1}\right)^{2}$ is the probability in the $r$ th step that Alice and Bob obtain coinciding outcomes in the measurements on the target pairs ( so only $p_{r-1} / 2$ of the pairs before the $r$ th step is surviving after the step ). Let us define the domains

$$
\begin{aligned}
\mathcal{D}_{a} & =\{a \in(0.5,1] ; a+b+c+d=1\}, \\
\mathcal{D}_{b} & =\{b \in(0.5,1] ; a+b+c+d=1\}, \\
\mathcal{D}_{c} & =\{c \in(0.5,1] ; a+b+c+d=1\}, \\
\mathcal{D}_{d} & =\{d \in(0.5,1] ; a+b+c+d=1\}, \\
\mathcal{D}_{a b} & =\mathcal{D}_{a} \cup \mathcal{D}_{b}, \\
\mathcal{D}_{c d} & =\mathcal{D}_{c} \cup \mathcal{D}_{d}, \\
\mathcal{D}_{a b c d} & =\mathcal{D}_{a} \cup \mathcal{D}_{b} \cup \mathcal{D}_{c} \cup \mathcal{D}_{d} .
\end{aligned}
$$

In what follows we will consider the case that an initial mixed state to be purified is in the applicable $\mathcal{D}_{a b c d}$ because any state $\rho \in \mathcal{D}_{a b c d}$ is distillable. It has been proved[12] that, for the Oxford protocol, an initial state in the domain $\mathcal{D}_{a b}$ will eventually be mapped to converge to the attractor $\{1,0,0,0\}$ representing the desired pure state $\left|\Phi^{+}\right\rangle\left\langle\Phi^{+}\right|$. While if the initial state is in the domain $\mathcal{D}_{c d}$, then it will be mapped to approach another attractor $\{0,0,1,0\}$, or the pure state $\left|\Psi^{+}\right\rangle\left\langle\Psi^{+}\right|$. In the end, according to ref.[7], using the QPA protocol, Alice and Bob will regain the desired pure state from any state $\rho \in \mathcal{D}_{a b c d}$ provided they first take efforts additional to the standard purification LOCC operations to transform the pure state $\left|\Psi^{+}\right\rangle\left\langle\Psi^{+}\right|$, or $\left|\Phi^{-}\right\rangle\left\langle\Phi^{-}\right|$, into the desired state $\left|\Phi^{+}\right\rangle\left\langle\Phi^{+}\right|$if the input state is in the domain $\mathcal{D}_{c d}$. Meanwhile, such efforts also have meaningful implication as if the QPA is considered to be combined with the hashing protocol[5, 6] to improve its output yield. These tedious transformations cannot be avoided even when the input state is already in the domain $\mathcal{D}_{a b}$, because Alice and Bob initially do not have an idea about whether the input state is exactly in the domain $\mathcal{D}_{a b}$ or $\mathcal{D}_{c d}$. For example, if the input state has the element $c_{0}=0.7$, then Alice and Bob should transform the state $\left|\Psi^{+}\right\rangle\left\langle\Psi^{+}\right|$into $\left|\Phi^{+}\right\rangle\left\langle\Phi^{+}\right|$before the purification procedure so that the mixed state in turn will have the element $a_{0}=0.7$. 
As another example, if Alice and Bob choose $\theta=\pi / 2$ and $\phi=0$, then they have the operator

$$
U(\pi / 2,0)=\mathbf{X H}=\frac{1}{\sqrt{2}}\left[\begin{array}{cc}
1 & -1 \\
1 & 1
\end{array}\right],
$$

where $\mathbf{X}$ is quantum NOT gate and $\mathbf{H}$ is the Hadamard transformation. Accordingly, in this case, the recurrence scheme is described by

$$
\begin{aligned}
& a_{r}=\frac{a_{r-1}^{2}+c_{r-1}^{2}}{p_{r-1}}, b_{r}=\frac{2 b_{r-1} d_{r-1}}{p_{r-1}}, \\
& c_{r}=\frac{b_{r-1}^{2}+d_{r-1}^{2}}{p_{r-1}}, d_{r}=\frac{2 a_{r-1} c_{r-1}}{p_{r-1}}, \text { for } \theta=\pi / 2, \phi=0,
\end{aligned}
$$

where $p_{r-1}=\left(a_{r-1}+c_{r-1}\right)^{2}+\left(b_{r-1}+d_{r-1}\right)^{2}$. It should be mentioned here that the relations $(7)$ can also be resulted from the utility of Hadamard transformation only, i.e., $U=\mathbf{H}$, but this transformation does not belong to the $\mathrm{SU}(2)$ operator defined in (2). Although the analytical convergency in the recurrence scheme (7) has not yet been proved, we find that an initial state in some domain $\mathcal{D}_{u} \subset \mathcal{D}_{a b c d}$, which is not yet defined, will be mapped to approach the periodic attractor representing a state interchanging step by step between $\{0.5,0,0,0.5\}$ and $\{0.5,0$, $0.5,0\}$, while a state in the domain $\mathcal{D}_{u}^{c}$, where $\mathcal{D}_{u}^{c} \cup \mathcal{D}_{u}=\mathcal{D}_{a b c d}$, will be mapped to converge to the fixed attractor $\{1,0,0,0\}$, as wanted. For example, one can easily check to see that the initial state $\{0.1,0.2,0.6,0.1\}$ will be mapped to converge to the fixed attractor but the initial state $\{0.2,0.1,0.6,0.1\}$, on the other hand, will be mapped to approach the mentioned periodic attractor. So a protocol in which the operator XH is used, unlike the QPA protocol, will not guarantee to purify pure maximally entangled pairs.

In this work, we call a protocol a one-map protocol if Alice and Bob each uses only one single local operator in all the purification LOCC operations, such as the IBM and Oxford protocols. From the above examples we realize that if only the standard purification LOCC operations are implemented, all one-map protocols will encounter the same situation that there is always another attractor in addition to the desired one, $\{1,0,0,0\}$, for a state $\rho \in \mathcal{D}_{a b c d}$ to be mapped to converge to. This situation thus becomes the ultimate limitation for the one-map algorithm. Therefore, in this work, we will present a viewpoint of hybrid maps for a purification protocol and show the fixed state $\{1,0,0,0\}$ can be the only attractor for an initial state $\rho \in \mathcal{D}_{a b c d}$ to be mapped to approach. The simple idea can be interpreted briefly. If we have known a one-map protocol, say, controlled by $\theta_{1}$ and $\phi_{1}$, in which a state $\rho$ belonging to some defined domain $\mathcal{D}_{1}\left(\subset \mathcal{D}_{a b c d}\right)$ can be mapped to approach the fixed attractor $\{1,0,0$, $0\}$, then all we have to do is to find another map, controlled by $\theta_{0}$ and $\phi_{0}$, in which a state $\rho \in \mathcal{D}_{a b c d}$ will be mapped on to a subdomain of the defined $\mathcal{D}_{1}$. This kind of protocol is what we call a two-map protocol, which can ensure Alice and Bob to regain the desired pure state $\left|\Phi^{+}\right\rangle\left\langle\Phi^{+}\right|$all by using the standard purification LOCC operations.

For the idea we have just presented, the most difficult task is the definition of the domain $\mathcal{D}_{1}$. Fortunately, Macchiavello[12] has defined the domain $\mathcal{D}_{1}$ for the QPA protocol, in which $\mathcal{D}_{1}=\mathcal{D}_{a b}$, as defined in (5). Therefore the QPA protocol is so far the most convenient one-map 
protocol to be improved by our idea. As to the one-map protocol described in (7), on the contrast, no definition of the corresponding $\mathcal{D}_{1}$ have been proved. A concrete example of our idea, however, will utilize these two one-map protocols. That is, in this example the option $\theta_{1}=\pi / 2$ and $\phi_{1}=\pi / 2$ will be chosen and accordingly the choice $\theta_{0}=\pi / 2$ and $\phi_{0}=0$ follows. We begin with the derivation of $\left(1-2 a_{r}\right)$ and $\left(1-2 c_{r}\right)$ for $\theta_{0}=\pi / 2$ and $\phi_{0}=0$. According to (7), we have

$$
1-2 a_{r}=\frac{\left(1-2 a_{r-1}\right)\left(1-2 c_{r-1}\right)}{p_{r-1}}, 1-2 c_{r}=\frac{\left(1-2 b_{r-1}\right)\left(1-2 d_{r-1}\right)}{p_{r-1}},
$$

for arbitrary positive integer $r$. It is now clear to find that, since $p_{r-1}>0$, when $a_{0}>1 / 2$ or $c_{0}>1 / 2$, then after the first purification LOCC operation we have $a_{1}>1 / 2$, while as $b_{0}>1 / 2$ or $d_{0}>1 / 2$, then we in turn have $c_{1}>1 / 2$, which consequently implies $a_{2}>1 / 2$ after the second purification LOCC operation. As a result, we know by now that using the one-map protocol (7), we can always in two steps map an initial state $\rho \in \mathcal{D}_{a b c d}$ on to the domain $\mathcal{D}_{a}$, which is exactly a subdomain of $\mathcal{D}_{1}\left(=\mathcal{D}_{a b}\right)$ for the standard QPA protocol. Now, we have come to the two-map protocols we wish to present in this work. Using this two-map protocol(symbolized by TM1), Alice and Bob have an agreement that in the first two steps of the purification procedure, they will apply the operators $U(\pi / 2,0)$ and $U^{*}(\pi / 2,0)$, respectively, to map a state $\rho \in \mathcal{D}_{a b c d}$ on to the domain $\mathcal{D}_{a}=\{a \in(0.5,1], a+b+c+d=1\}$, and then they will apply the standard QPA operators $U(\pi / 2, \pi / 2)$ and $U^{*}(\pi / 2, \pi / 2)$ to purify the surviving state to the desired state $\left|\Phi^{+}\right\rangle\left\langle\Phi^{+}\right|$in the rest purification LOCC operations. Interestingly, an alternative two-map protocol (symbolized by TM2) can be used as well, in which the operators $U(\pi / 2,0)$ and $U^{*}(\pi / 2,0)$ are applied only at the second purification LOCC operation, since after the first LOCC operation, in which the QPA operators $U(\pi / 2, \pi / 2)$ and $U^{*}(\pi / 2, \pi / 2)$ are used, the state has been mapped on to the domain $\mathcal{D}_{a c}[12]$.

Apparently, our protocols TM1 and TM2 are composed of only the standard purification LOCC operations, without using any additional local operations and classical communications in transforming the mixed state into a Werner state, as needed in the IBM protocol, or transforming one of the Bell states whose fidelity is the largest into the desired pure state $\left|\Phi^{+}\right\rangle$ in advance of the Oxford operations. Therefore the fewest operations are required in our purification algorithms, as compared with the IBM and Oxford protocols. Furthermore, when comparing the output yields and the fidelities (or purities) produced by the IBM, the Oxford, and our two-map protocols, we find the protocol TM1 can provide better yields and fidelities than the Oxford protocol (which performs better than the IBM protocol), while the protocol TM2 can perform almost equally to the Oxford protocol, although this is not the primary purpose of this work. In our numerical simulations, the yield, or the fraction of the surviving pairs, defined by $Y_{r}=p_{0} p_{1} \ldots p_{r-1}\left(2^{-r}\right)$, where $r$ denotes the iteration number, was first computed up to $r=10$ for each input state to be purified. The variations of the yield as functions of the initial fidelity $a_{0}$ are shown in Fig. 2, in which (and also in the following figures) each yield (and each purity) was the average value computed over ten thousand random states possessing the same initial fidelity. The corresponding purities after the ten iterations are also shown in Fig. 2. It is shown that although, after the ten iterations, the resulted purities produced by using the Oxford, TM1, and TM2 are high, the yields of them are rather poor, especially when 
Figure 2: The variations of the yield and the comparing purity (in the inserted diagram) at ten times of the recurrence method.

the initial fidelity is close to $1 / 2$. The yield, however, can be further improved by combining the recurrence method with the hashing protocol[5, 6] as long as the purity is high enough (e.g. higher than 0.8107 for a Werner state) when the recurrence scheme is performed in only a few iterations. In Fig. 3 we show the yields $Y_{5}$ and the correspond purities $a_{5}$ produced by the Oxford and the TM1 protocols after five iterations, respectively. This figure shows that when the initial fidelities are greater than some specific values near $1 / 2$ for both cases (of course the specific fidelities can be lowered if the iteration is increased), the hashing protocols then are applicable after the five iterations in running the recurrence schemes. Fig.3 shows that after the five iterations, the surviving fraction $Y_{5, T M 1}$ and the corresponding purity $a_{5, T M 1}$, produced by the the TM1 protocol are slightly higher than the surviving fraction $Y_{5, O x}$ and the purity $a_{5, O x}$, which are resulted from using the Oxford protocol. The slight differences in $Y_{5}$ and $a_{5}$, however, can induce significant difference between the improved yields when the hashing protocol is switched on after the five iterations. The evidence can be seen in Fig. 4, in which both the improved yields $Y_{5, T M 1}^{\prime}$ and $Y_{5, O x}^{\prime}$ and the ratio of the improved yields $\left(Y_{5, T M 1}^{\prime} / Y_{5, O x}^{\prime}\right)$ as functions of the initial fidelity are shown; the improved yield is defined by $Y_{r}^{\prime}=Y_{r}\left(1-S\left(\rho_{r}\right)\right)$, where $\rho_{r}$ is the von Neumann entropy of the surviving mixed state $\rho_{r}$. It is clearly shown in Fig. 4 that the ratio $Y_{5, T M 1}^{\prime} / Y_{5, O x}^{\prime}$ becomes greater as the initial fidelity is closer to $1 / 2$.

To summarize, in the recurrence scheme of a one-map entanglement purification protocol, the nonlinear behavior of the four Bell-diagonal elements of the density matrix representing 
Figure 3: The variations of the yield and the comparing purity (in the inserted diagram) at five times of the recurrence method.

the mixed state to be purified reveals that there is always another attractor other than the desired fixed attractor. This indicates that not all the distillable input state can be purified to the desired maximally entangled pure state all by the standard purification LOCC operations in a one-map protocol. Therefore some tedious efforts additional to the purification LOCC operations are needed in using the typical IBM and Oxford protocols to purify a desired pure state from any distillable state. The proposed two-map purification protocols TM1 and TM2, on the contrast, can guarantee that all the distillable input states can be purified to the desired pure state all by the standard purification LOCC operations. That an entanglement purification can be accomplished all by the standard purification LOCC operations is crucially important to a significant improvement for the purification process. By such improvement, we then do not have to identify the mixed state and consequently do not consume any pairs before the purification LOCC operations. The proposed two-map protocols perform better than the onemap IBM and Oxford protocols in the sense that they require the least operation times in yielding a same amount of useful EPR pairs. Surprisingly, the protocol TM1 is found able to induce higher yields and purities than the Oxford protocol. This is crucially important as the hashing protocol is combined with the recurrence algorithm to improve the output yield. The proposed two-map protocols, however, like the standard IBM and Oxford protocols, should be implemented if the initial state possesses a fidelity very close to $1 / 2$ only after enhancing the state's fidelity. For example, it has been shown[13] that only inseparable two-qubit state with 
Figure 4: The variations of the improved yields $Y_{5, T M 1}^{\prime}$ and $Y_{5, O x}^{\prime}$ and the comparing ratio $\left(Y_{5, T M 1}^{\prime} / Y_{5, O x}^{\prime}\right)$ (in the inserted diagram).

"free" entanglement, however small, can be distillable to a pure form by using local filtering[14, 15] to enhance the state's fidelity first. An interaction with the environment[16] can even be allowed to enhance the fidelity of a quantum teleportation. The fidelity enhancement, however, is not an issue to be concerned with in this work.

\section{References}

[1] C. H. Bennett, G. Brassard, C. Crepeau, R. Jozsa, A. Peres, and W. K. Wootters, "Teleporting an unknown quantum state via dual classical and Einstein-Podolsky-Rosen channels," Phys. Rev. Lett., vol. 70, no. 13, pp. 1895-1899, 1993.

[2] B. Schumacher, "Quantum coding", Phys. Rev. A, vol. 51, no. 4, pp. 2738-2747, 1995.

[3] R. Jozsa and B. Schumacher, "A New Proof of the Quantum Noiseless Coding Theorem," J. Modern Optics, vol. 41, pp. 2343-2350, 1994.

[4] A. K. Ekert, "Quantum cryptography based on Bell's theorem," Phys. Rev. Lett., vol. 67, no. 6, pp. 661-663 , 1991. 
[5] C. H. Bennett, D. P. DiVincenzo, J. A. Smolin, and W. K. Wootters, "Mixed-state entanglement and quantum error correction," Phys. Rev. A, vol. 54, no. 5, pp. 3824-3851, 1996.

[6] C. H. Bennett, G. Brassard, S. Popescu, B. Schumacher, J. A. Smolin, and W. K. Wootters, "Purification of Noisy Entanglement and Faithful Teleportation via Noisy Channels," Phys. Rev. Lett., vol. 76, no.5, pp. 722-725,1996.

[7] D. Deutsch, A. Ekert, R. Jozsa, C. Macchiavello, S. Popescu, and A. Sanpera, "Quantum Privacy Amplification and the Security of Quantum Cryptography over Noisy Channels," Phys. Rev. Lett., vol. 77, no. 13, pp. 2818-2821,1996.

[8] R. F. Werner, "Quantum states with Einstein-Podolsky-Rosen correlations admitting a hidden-variable model", Phys. Rev. A, vol. 40, no.8, pp. 4277- 4281, 1989.

[9] E. Maneva and J. Smolin, "Improved two-party and multi-party purification protocols," LANL e-print quant-ph/0003099.

[10] N. Metwally, "More efficient entanglement purification," Phys. Rev. A, vol. 66, no.5, pp. 054302-5, 2002.

[11] J. Dehaene, M. Van den Nest, B. De Moor, and F. Verstraete, "Local permutations of products of Bell states and entanglement distillation," Phys. Rev. A, vol. 67, no. 2, pp. 022310-6, 2003.

[12] C. Macchiavello, "On the analytical convergence of the QPA procedure," Phys. Lett. A, vol. 246, pp. 385-388, 1998.

[13] M. Horodecki, P. Horodecki, and R. Horodecki, "Inseparable Two Spin- 1 / 2 Density Matrices Can Be Distilled to a Singlet Form," Phys. Rev. Lett., vol. 78, no.4, pp. 574-577, 1997 ;

M. Horodecki, P. Horodecki, and R. Horodecki, "Mixed-State Entanglement and Distillation: Is there a "Bound" Entanglement in Nature?," Phys. Rev. Lett., vol. 80, no.24, pp. 5239-5242, 1998.

[14] N. Gisin, "Hidden quantum nonlocality revealed by local filters," Phys. Lett. A, vol. 210, pp. 151-156, 1996.

[15] C. H. Bennett, H. J. Bernstein, S. Popescu, and B. Schumacher, "Concentrating partial entanglement by local operations ," Phys. Rev. A, vol. 53, no. 4, pp. 2046-2052,1996.

[16] P. Badziag, M. Horodecki, P. Horodecki, and R. Horodecki, "Local environment can enhance fidelity of quantum teleportation," Phys. Rev. A., vol. 62, no. 1, pp. 012311-7, 2000. 
A

B

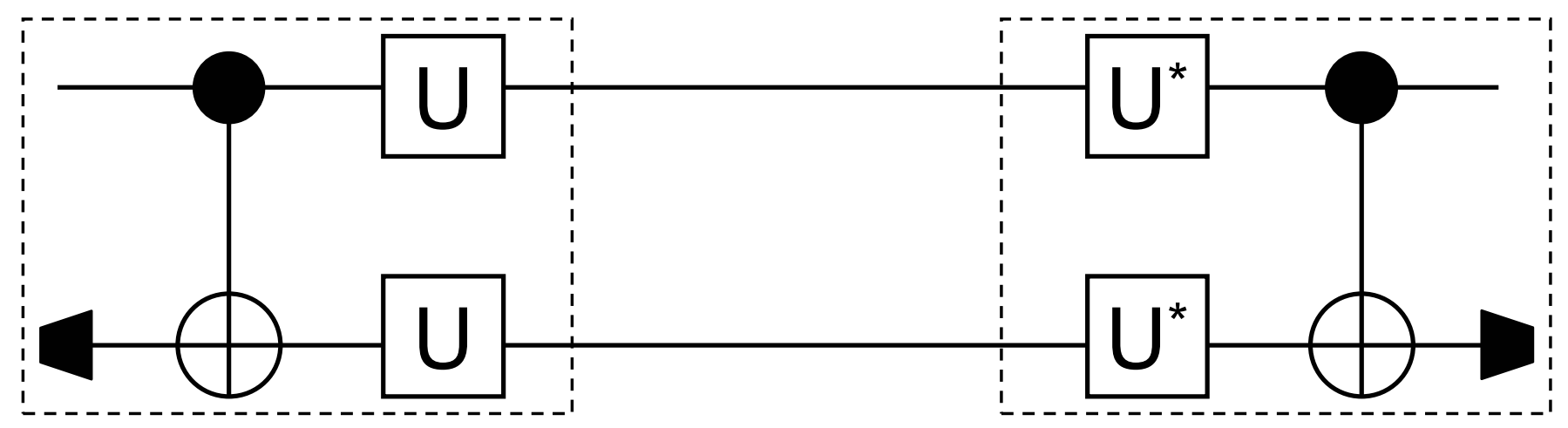




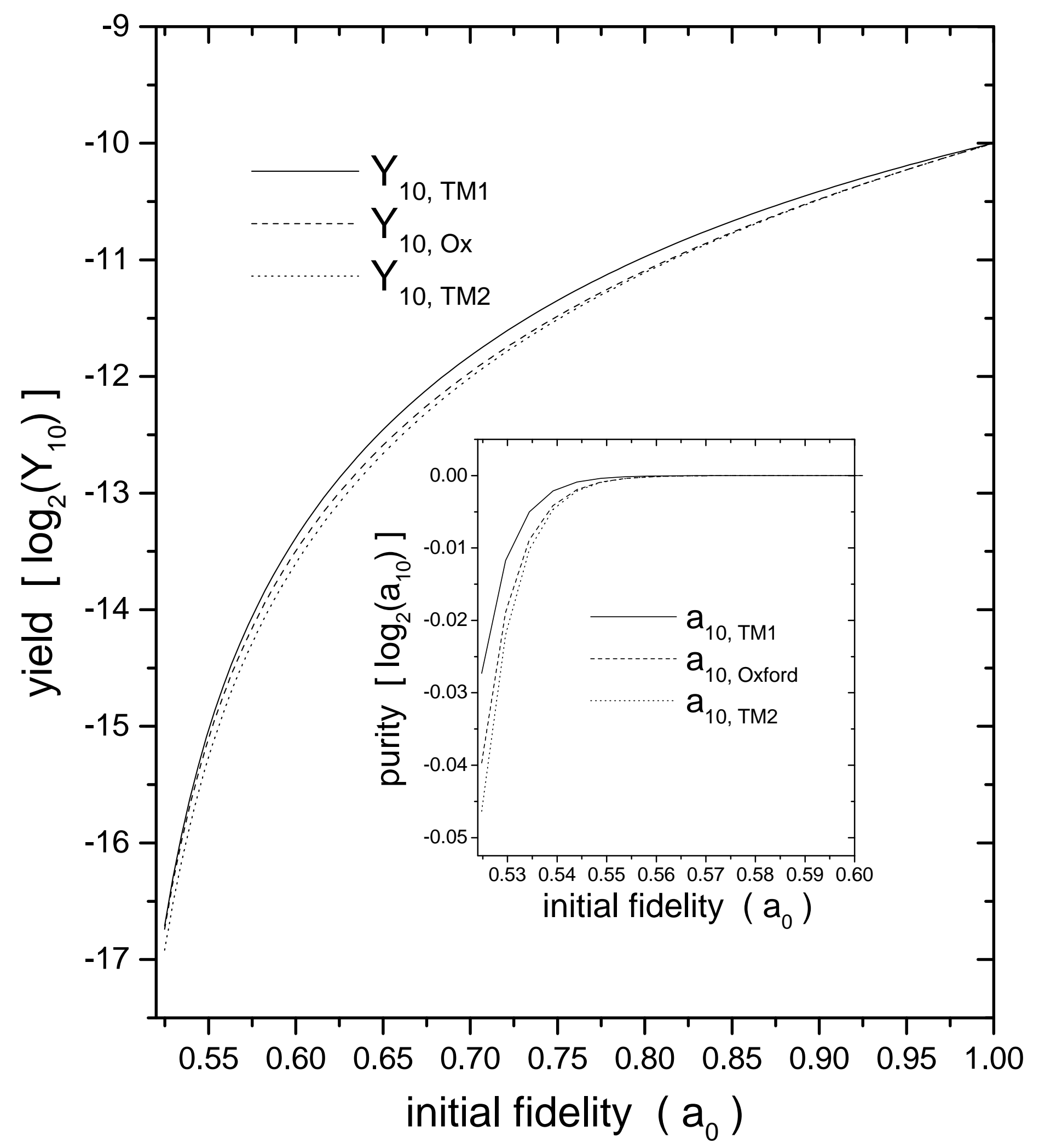




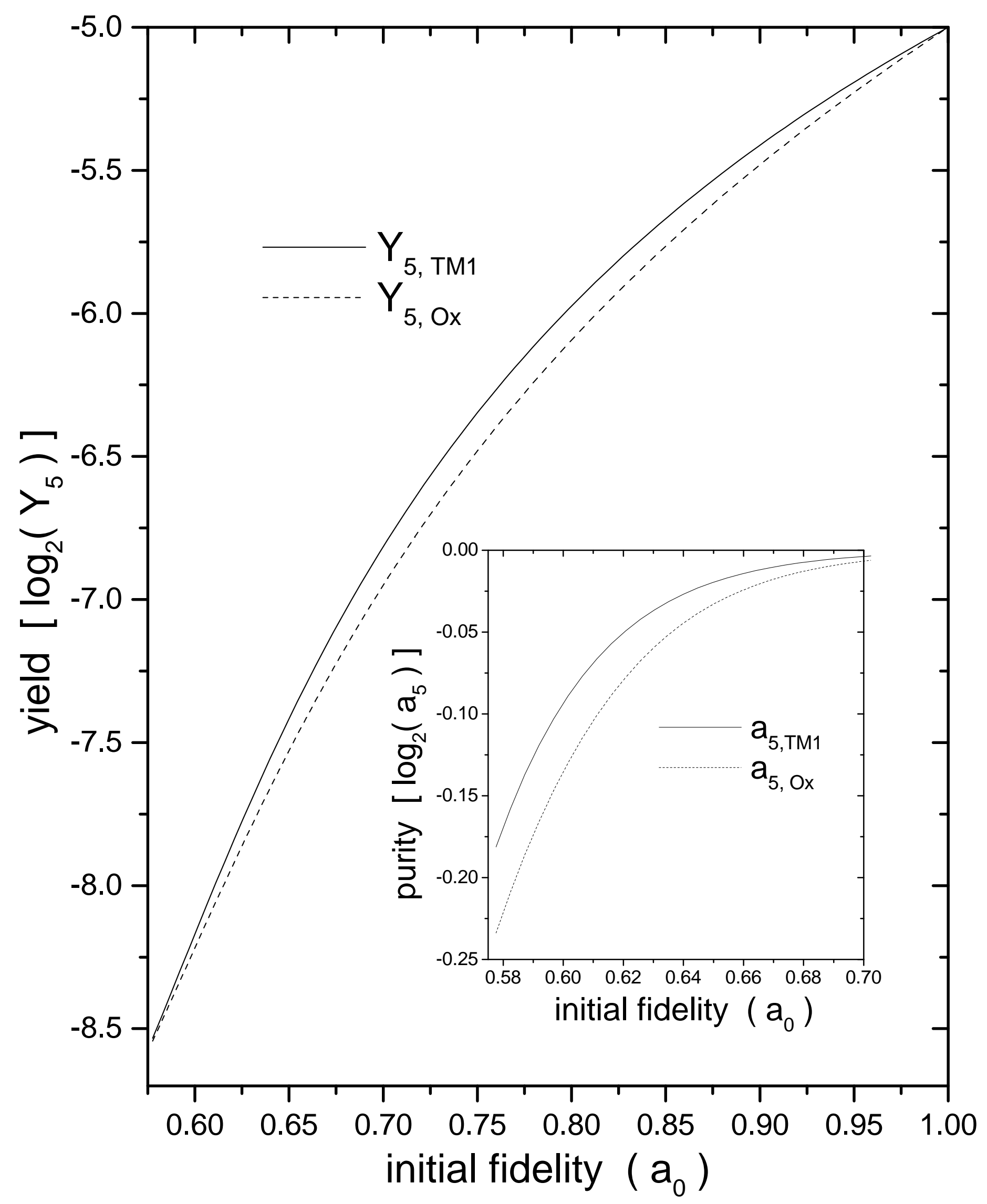




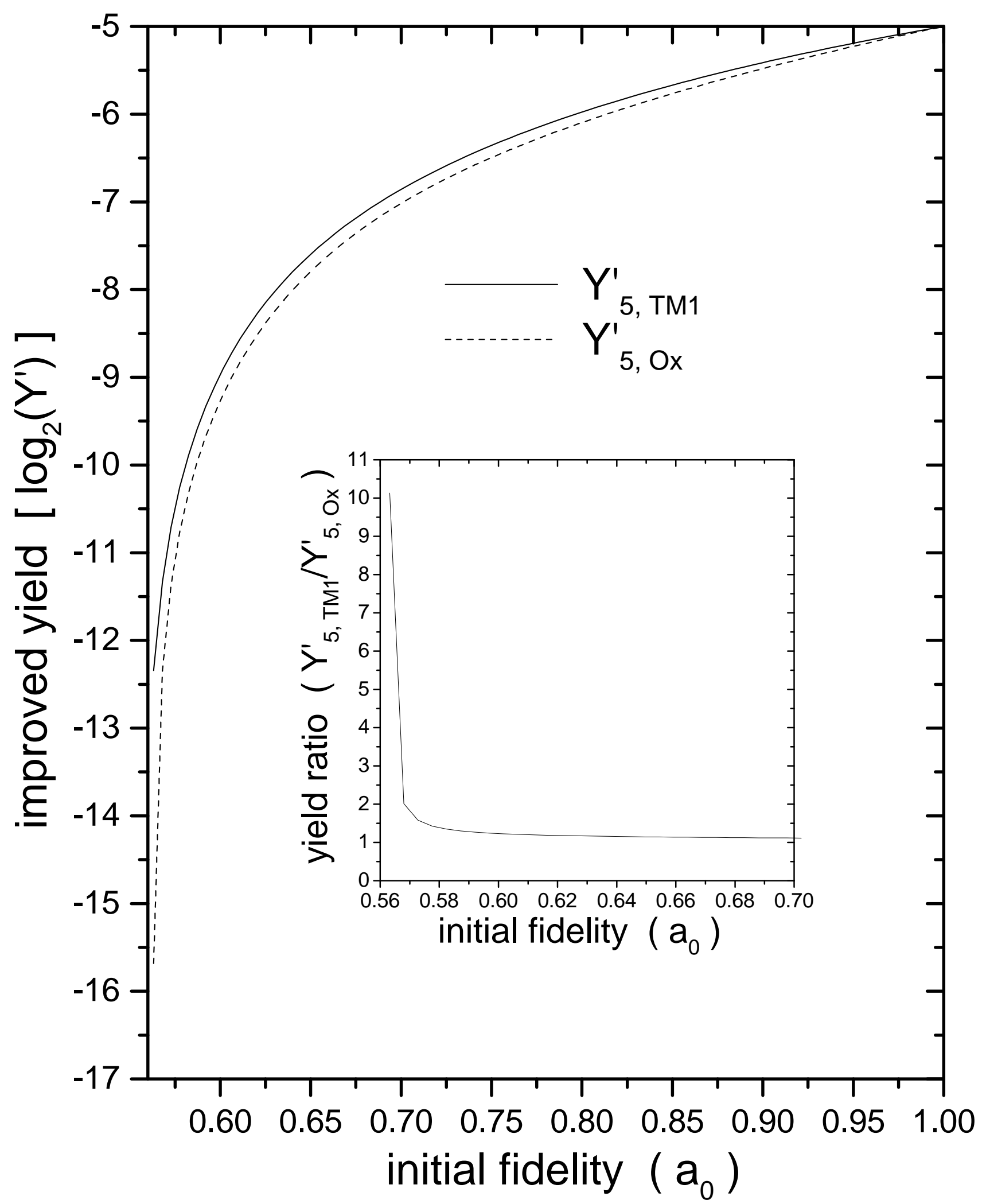

